\title{
Extension Fullness of the Categories of Gelfand-Zeitlin and Whittaker Modules*
}

\author{
Kevin COULEMBIER ${ }^{\dagger}$ and Volodymyr MAZORCHUK ${ }^{\ddagger}$ \\ $\dagger$ Department of Mathematical Analysis, Ghent University, Krijgslaan 281, 9000 Gent, Belgium \\ E-mail:coulembier@cage.ugent.be \\ URL: http://cage.ugent.be/ coulembier/ \\ ¥Department of Mathematics, Uppsala University, Box 480, SE-751 06, Uppsala, Sweden \\ E-mail: mazor@math.uu.se \\ URL: http://www2.math.uu.se/ mazor/
}

Received September 25, 2014, in final form February 20, 2015; Published online February 24, 2015 http://dx.doi.org/10.3842/SIGMA.2015.016

\begin{abstract}
We prove that the categories of Gelfand-Zeitlin modules of $\mathfrak{g}=\mathfrak{g l}_{n}$ and Whittaker modules associated with a semi-simple complex finite-dimensional algebra $\mathfrak{g}$ are extension full in the category of all $\mathfrak{g}$-modules. This is used to estimate and in some cases determine the global dimension of blocks of the categories of Gelfand-Zeitlin and Whittaker modules.
\end{abstract}

Key words: extension fullness; Gelfand-Zeitlin modules; Whittaker modules; Yoneda extensions; homological dimension

2010 Mathematics Subject Classification: 16E30; 17B10

\section{Introduction}

Homological invariants are useful technical tools in modern representation theory. As classification of all modules of a given (Lie) algebra is a wild problem in almost all non-trivial and interesting cases (see e.g. [5, 11, 19]), the usual "reasonable" setup for the study of representations of a given (Lie) algebra assumes some fixed subcategory of the category of all modules. Therefore, the problem to compare homological invariants for a given category and some of its subcategories is natural and important.

Given an Abelian category $\mathcal{A}$ and an Abelian subcategory $\mathcal{B}$ of $\mathcal{A}$ such that the natural inclusion $\mathcal{B} \hookrightarrow \mathcal{A}$ is exact, we say that $\mathcal{B}$ is extension full in $\mathcal{A}$ provided that the natural inclusion induces isomorphisms $\operatorname{Ext}_{\mathcal{B}}^{d}(M, N) \cong \operatorname{Ext}_{\mathcal{A}}^{d}(M, N)$ for all $M, N \in \mathcal{B}$ and all $d \geq 0$, see Subsection 2.2 for details (we write $X \in \mathcal{C}$ when $M$ is an object of some category $\mathcal{C}$ ). Extension fullness is a useful notion which allows one to freely transfer homological information between categories $\mathcal{A}$ and $\mathcal{B}$. Recently this concept of extension fullness has also been studied by Herman in [25], where it appears under the name entirely extension closed.

Motivated by the so-called Alexandru conjecture from [17, 22] (a part of which asserts extension fullness of certain categories in Lie theory), in our previous paper [7] we proved that the category $\mathcal{O}$ associated with a semi-simple complex finite-dimensional Lie algebra $\mathfrak{g}$ is extension full in the category of all weight $\mathfrak{g}$-modules, and that the thick version of $\mathcal{O}$ is extension full in the category of all $\mathfrak{g}$-modules. As a bonus, we determined the global dimension of the thick category $\mathcal{O}$ as well as projective dimensions of its simple objects.

\footnotetext{
${ }^{\star}$ This paper is a contribution to the Special Issue on New Directions in Lie Theory. The full collection is available at http://www.emis.de/journals/SIGMA/LieTheory2014.html
} 
Although category $\mathcal{O}$ is probably the most studied category of $\mathfrak{g}$-modules, there are several other natural and well-studied categories which have rather different flavor. One of them is the category $\mathscr{G} \mathscr{Z}$ of so-called Gelfand-Zeitlin ${ }^{1}$ modules, introduced in [13] for the algebra $\mathfrak{s l}_{3}(\mathbb{C})$, in [14] for the algebra $\mathfrak{g l}_{n}(\mathbb{C})$ and in [34] for orthogonal Lie algebras. The category $\mathscr{G} \mathscr{Z}$ can be seen as a generalization of $\mathcal{O}$ in the sense that it contains both $\mathcal{O}$ and the thick version of $\mathcal{O}$. The study of Gelfand-Zeitlin modules attracted considerable attention, see e.g. [12, 18, 20, 21, 26, 28, 33, 35, 36, 37, 44, 45, 46] and references therein. As far as we know, simple generic Gelfand-Zeitlin modules give the richest known family of simple $\mathfrak{g l}_{n}(\mathbb{C})$-modules. This family depends on $\frac{n(n+1)}{2}$ generic parameters. The first main result of this paper is the following statement proved in Section 3 (we refer to Sections 2 and 3 for more details):

Theorem A. The category $\mathscr{G} \mathscr{Z}$ is extension full in the category of all $\mathfrak{g l}_{n}$-modules.

The added difficulty of the category $\mathscr{G} \mathscr{Z}$ in comparison with thick category $\mathcal{O}$ in [7] is that $\mathscr{G} \mathscr{Z}$ is not a Serre subcategory generated by a well-known category which has enough projective objects (contrary to the relation between category $\mathcal{O}$ and thick category $\mathcal{O}$ ). Therefore to prove Theorem A we have to modify and strengthen the abstract results on extension fullness in [7]. Our arguments also heavily use some properties of Gelfand-Zeitlin modules established by Futorny and Ovsienko in [21].

Another big class of $\mathfrak{g}$-modules, where now $\mathfrak{g}$ is an arbitrary semi-simple Lie algebra with a fixed triangular decomposition $\mathfrak{g}=\mathfrak{n}_{-} \oplus \mathfrak{h} \oplus \mathfrak{n}_{+}$, is the class of so-called Whittaker modules introduced by Kostant in [29]. Simple Whittaker modules are simple $\mathfrak{g}$-modules on which the algebra $U\left(\mathfrak{n}_{+}\right)$acts locally finitely, see also [3] for a general Whittaker setup. These modules were studied in $[27,38,39,40,48]$ in the classical setup. Generalizations of these modules for (infinite-dimensional) Lie algebras and some related algebras attracted a lot of attention recently, see $[2,3,4,6,24,41,42,43]$ and references therein.

We define the category $\mathscr{W}$ of Whittaker modules of finite length and for this category we prove the next statement, which is our second main result (we refer to Section 4 for more details):

Theorem B. The category $\mathscr{W}$ is extension full in the category of all $\mathfrak{g}$-modules.

Using adjunction, the study of $\mathscr{W}$ reduces to the study of locally finite modules over a certain Noetherian algebra. An added difficulty compared to the case of the category $\mathscr{G} \mathscr{Z}$ is that a module in $\mathscr{W}$ does not decompose into a direct sum of finite-dimensional $U\left(\mathfrak{n}_{+}\right)$-modules despite the fact that the action of $U\left(\mathfrak{n}_{+}\right)$is locally finite. Moreover, a module in $\mathscr{W}$ is not always finitely generated over $U\left(\mathfrak{n}_{+}\right)$. To be able to prove extension fullness, we crucially depend on a result of Donkin and Dahlberg from the 80's (see [10] and [8]) asserting that essential extensions of locally finite modules over solvable finite-dimensional Lie algebras are locally finite.

An advantage of the result in Theorems A and B is that it allows to replace the calculation of extensions in a category without projective objects by the calculation of extensions in a category with enough projective objects. In particular, as a consequence we obtain that the global dimension of the categories $\mathscr{G} \mathscr{Z}$ and $\mathscr{W}$ equals $\operatorname{dim} \mathfrak{g}$. As both category $\mathcal{O}$ and its thick version are full subcategories in $\mathscr{G} \mathscr{Z}$ or $\mathscr{W}$ (thick category $\mathcal{O}$ is even a Serre subcategory, as defined in [49, Subsection 10.3.2]), combining Theorems A and B with the results of [7] gives, in particular, a lower bound on the projective dimension of simple highest weight and Verma modules in the categories $\mathscr{G} \mathscr{Z}$ and $\mathscr{W}$.

The paper is organized as follows: in Section 2 we prove some preliminary homological algebra statements, Section 3 deals with the case of Gelfand-Zeitlin modules and Section 4 is devoted to the case of Whittaker modules.

\footnotetext{
${ }^{1}$ The surname Zeitlin is spelled Ceitlin in Russian. It appeared in different transliterations in Latin script, in particular, as Cetlin, Zetlin, Tzetlin and Tsetlin. However, it seems that the origin of this surname is the German word "Zeit" which justifies our present version.
} 


\section{Extension full subcategories}

\subsection{Extensions in Abelian categories}

Let $\mathcal{A}$ be an Abelian category and $M, N \in \mathcal{A}$. Recall that, for $d \in \mathbb{Z}_{\geq 0}$, the set $\operatorname{Ext}_{\mathcal{A}}^{d}(M, N)$ of degree $d$ extensions from $M$ to $N$ is defined as the set of equivalence classes of exact sequences

$$
0 \longrightarrow N \longrightarrow X_{1} \longrightarrow X_{2} \longrightarrow \cdots \longrightarrow X_{d} \longrightarrow M \longrightarrow 0
$$

where all objects and all morphisms are in $\mathcal{A}$, modulo the minimal equivalence relation which contains the binary relation given by existence of a commutative diagram

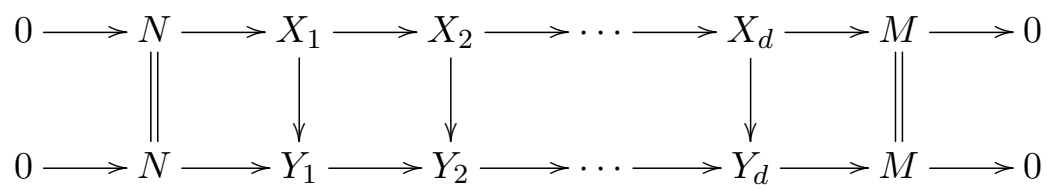

The set $\operatorname{Ext}_{\mathcal{A}}^{d}(M, N)$ has the natural structure of an Abelian group via the Baer sum. If $\mathcal{A}$ is $\mathbb{k}$-linear for some field $\mathbb{k}$, then $\operatorname{Ext}_{\mathcal{A}}^{d}(M, N)$ has the structure of a $\mathbb{k}$-vector space. We refer to [49, Section 3.4] for further information and details.

For $M \in \mathcal{A}$, the projective dimension $\operatorname{proj} \cdot \operatorname{dim}(M)$ of $M$ is defined as the maximal $d$ such that there is $N \in \mathcal{A}$ with $\operatorname{Ext}_{\mathcal{A}}^{d}(M, N) \neq 0$. If such maximal $d$ does not exist, then $\operatorname{proj} \cdot \operatorname{dim}(M):=$ $\infty$. Dually one defines the injective dimension inj. $\operatorname{dim}(N)$ for $N \in \mathcal{A}$. The global dimension $\operatorname{gl} \operatorname{dim}(\mathcal{A}) \in \mathbb{Z}_{\geq 0} \cup\{\infty\}$ is the supremum of projective dimensions taken over all objects in $\mathcal{A}$. The global dimension coincides with the supremum of injective dimensions taken over all objects in $\mathcal{A}$ (see [51, Lemma 5.11.11]).

\subsection{Extension full subcategories}

Let $\mathcal{A}$ be an Abelian category and $\mathcal{B}$ a full Abelian subcategory of $\mathcal{A}$ in the sense that the Abelian structure of $\mathcal{B}$ is inherited from $\mathcal{A}$. In particular, the natural inclusion functor $\iota: \mathcal{B} \rightarrow \mathcal{A}$ is exact. Then, for every $M, N \in \mathcal{B}$ and every $d \in \mathbb{Z}_{\geq 0}$, the functor $\iota$ induces homomorphisms

$$
\iota_{M, N}^{d}: \operatorname{Ext}_{\mathcal{B}}^{d}(M, N) \rightarrow \operatorname{Ext}_{\mathcal{A}}^{d}(M, N)
$$

of Abelian groups. In general, these homomorphisms $\iota_{M, N}^{d}$ are neither injective nor surjective.

We say that $\mathcal{B}$ is extension full in $\mathcal{A}$ provided that $\iota_{M, N}^{d}$ are bijective for all $d \in \mathbb{Z}_{\geq 0}$ and for all $M, N \in \mathcal{B}$. We refer to [7, Section 2] for details.

Let $0 \rightarrow K \rightarrow M \rightarrow N \rightarrow 0$ be a short exact sequence in $\mathcal{B}$. Then, for $Q \in \mathcal{B}$, application of $\operatorname{Hom}_{\mathcal{B}}\left(Q,,_{-}\right)$and $\operatorname{Hom}_{\mathcal{A}}\left(Q,,_{-}\right)$to this short exact sequence produces the usual long exact sequences in homology for the categories $\mathcal{B}$ and $\mathcal{A}$, respectively. Moreover, the homomorphisms $\iota_{Q,-}^{d}$ give rise to a homomorphism between these long exact sequences. A similar statement is true for $\operatorname{Hom}_{\mathcal{B}}(-, Q)$ and $\operatorname{Hom}_{\mathcal{A}}(-, Q)$.

\subsection{Checking extension fullness}

In this section we formulate and prove three propositions which will be useful for our study of extension fullness later in the paper. The following statement is a modification of [7, Lemma 4] which also allows for a somewhat stronger formulation.

Proposition 1. Let $\mathcal{A}$ be an Abelian category and $\mathcal{B}$ a Serre subcategory of $\mathcal{A}$. Assume that $\mathcal{B}$ contains a full subcategory $\mathcal{B}_{0}$ with the following properties:

(a) Every object in $\mathcal{B}$ is a quotient of an object in $\mathcal{B}_{0}$. 
(b) The homomorphisms $\iota_{M, N}^{d}$ are bijective for all $d \in \mathbb{Z}_{\geq 0}, M \in \mathcal{B}_{0}$ and $N \in \mathcal{B}$.

Then $\mathcal{B}$ is extension full in $\mathcal{A}$.

Proof. Our proof is similar to that of [7, Lemma 4]. We prove the statement by induction on $d$. Since $\mathcal{B}$ is assumed to be a Serre subcategory of $\mathcal{A}$, it is clear that $\iota_{Q, N}^{0}$ and $\iota_{Q, N}^{1}$ are isomorphisms for all $Q, N \in \mathcal{B}$.

To prove the induction step, for $Q \in \mathcal{B}$ consider a short exact sequence

$$
0 \rightarrow K \rightarrow M \rightarrow Q \rightarrow 0
$$

with $M \in \mathcal{B}_{0}$ and $K \in \mathcal{B}$, which exists by condition (a). Applying $\operatorname{Hom}_{\mathcal{B}}(-, N)$ and $\operatorname{Hom}_{\mathcal{A}}(-, N)$ gives for each $d$ the following commutative diagram with exact rows:

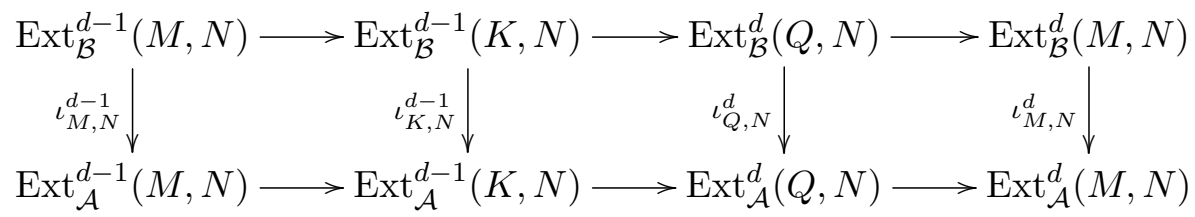

Now, $\iota_{M, N}^{d-1}$ and $\iota_{M, N}^{d}$ are isomorphisms by condition (b) and from the induction step we have that $\iota_{K, N}^{d-1}$ is a monomorphism. The injective four lemma (by which we mean the statement of [31, Lemma I.3.3(i)]) therefore implies that $\iota_{Q, N}^{d}$ is a monomorphism.

It remains to show that $\iota_{Q, N}^{d}$ is an epimorphism. For this we consider the following commutative diagram with exact rows:

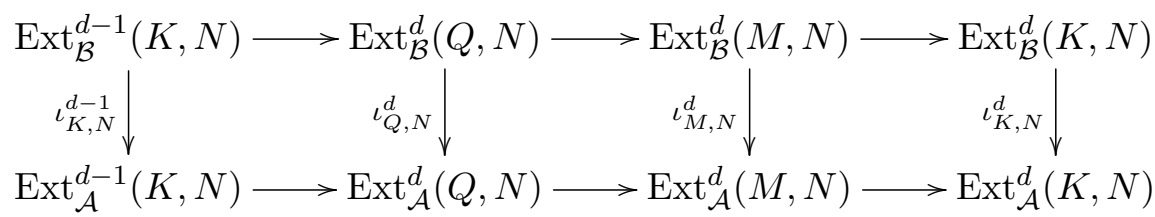

As $\iota_{K, N}^{d-1}$ is a bijection by the induction step, $\iota_{M, N}^{d}$ is a bijection by condition (b) and $\iota_{K, N}^{d}$ is a monomorphism by the previous paragraph, the surjective four lemma (by which we mean the statement of [31, Lemma I.3.3(ii)]) implies that $\iota_{Q, N}^{d}$ is an epimorphism. This completes the proof.

The proof of the following proposition is dual to that of [7, Corollary 5] concerning acyclicness (see [49, Subsection 2.4.3]) of projective objects.

Proposition 2. Let $\mathcal{A}$ be an Abelian category and $\mathcal{B}$ a full Abelian subcategory of $\mathcal{A}$ and assume that they both have enough injective objects. If every injective object in $\mathcal{B}$ is acyclic for the functor $\operatorname{Hom}_{\mathcal{A}}\left(K,{ }_{-}\right)$for any $K \in \mathcal{B}$, then $\mathcal{B}$ is extension full in $\mathcal{A}$.

Proof. Consider $K \in \mathcal{B}$ fixed. We need to prove that the functor $\operatorname{Ext}_{\mathcal{A}}^{d}\left(K,{ }_{-}\right)$, when restricted to the category $\mathcal{B}$, is isomorphic to the functor $\operatorname{Ext}_{\mathcal{B}}^{d}\left(K,{ }_{-}\right)$. We have the obvious isomorphism

$$
\operatorname{Hom}_{\mathcal{B}}\left(K,_{-}\right) \cong \operatorname{Hom}_{\mathcal{A}}\left(K,{ }_{-}\right) \circ \boldsymbol{\iota},
$$

of functors from the category $\mathcal{B}$ to the category Sets.

By assumption, the exact functor $\iota$ maps injective objects in $\mathcal{B}$ to injective objects in $\mathcal{A}$. Injective objects in $\mathcal{A}$ are acyclic for the functor $\operatorname{Hom}_{\mathcal{A}}\left(K,{ }_{-}\right)$, that is for all such objects $I$ we have $\operatorname{Ext}_{\mathcal{A}}^{d}(K, I)=0$ for all $d>0$. The classical Grothendieck spectral sequence, see [49, Section 5.8], therefore implies the statement. 
Let us fix the following notation: for an associative algebra $A$ over a field $\mathbb{k}$ denote by $A$-Mod the category of all $A$-modules. We also denote by $A$-mod the full subcategory of $A$-Mod consisting of all finitely generated modules. We denote by $A$-lfmod the full subcategory of $A$-Mod consisting of all modules on which the action of $A$ is locally finite. Finally, we denote by $A$-fmod the full subcategory of $A$-mod consisting of all finite-dimensional modules.

Proposition 3. Consider an associative algebra $A$, a full Abelian subcategory $\mathcal{A}$ in $A$-Mod, and a full Abelian subcategory $\mathcal{B}$ of $\mathcal{A}$. Assume that these data satisfy the following conditions:

(a) $\mathcal{B}$ is a Serre subcategory of $A$-Mod.

(b) For every surjective morphism $\alpha: M \rightarrow N$, with $M \in \mathcal{A}$ and $N \in \mathcal{B}$, there is $a \in \mathcal{B}$ and an injective morphism $\beta: Q \hookrightarrow M$ such that the composition $\alpha \circ \beta: Q \rightarrow N$ is surjective.

Then $\mathcal{B}$ is extension full in $\mathcal{A}$.

Proof. For $d>0$ let

$$
0 \rightarrow M \rightarrow X_{1} \rightarrow X_{2} \rightarrow \cdots \rightarrow X_{d-1} \rightarrow X_{d} \rightarrow N \rightarrow 0
$$

and

$$
0 \rightarrow M \rightarrow Y_{1} \rightarrow Y_{2} \rightarrow \cdots \rightarrow Y_{d-1} \rightarrow Y_{d} \rightarrow N \rightarrow 0
$$

be exact sequences in $\mathcal{B}$. Assume that there is a commutative diagram in $\mathcal{A}$ with exact rows as follows:

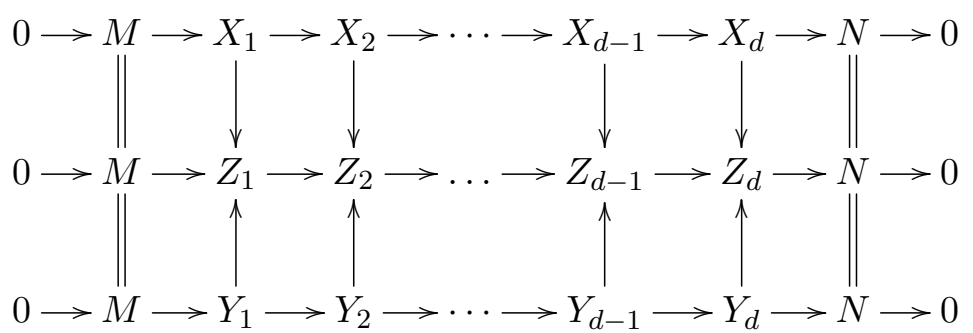

For $i=1,2, \ldots, d$, let $Q_{i}$ denote the submodule of $Z_{i}$ generated by the images of $Y_{i}$ and $X_{i}$. Since $\mathcal{B}$ is a Serre subcategory of $A$-Mod, we have that $Q_{i}$ belongs to $\mathcal{B}$. Then diagram (1) restricts to the commutative diagram

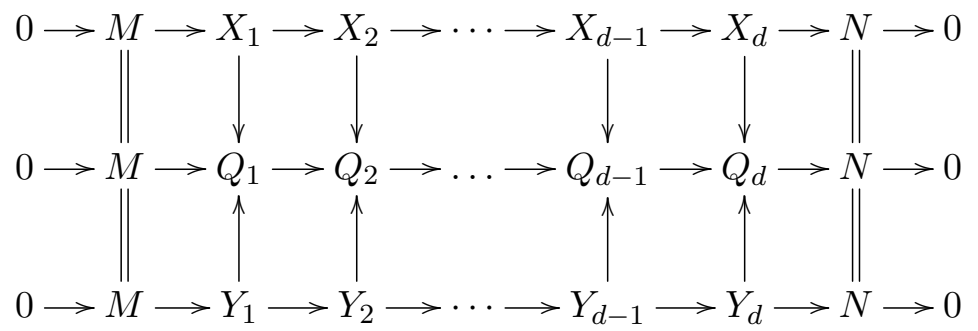

in which the complex in the second row might be not exact. By assumption, there is a submodule in $Z_{d}$ which surjects onto $N$ and is in $\mathcal{B}$. The sum of that submodule with $Q_{d}$ is also in $\mathcal{B}$ since $\mathcal{B}$ is a Serre subcategory of $A$-Mod. We denote this resulting submodule by $T_{d}$. The kernel $K_{d}$ of the surjection $T_{d} \rightarrow N$ is also in $\mathcal{B}$ as $\mathcal{B}$ is Abelian. By the same reasoning there is a submodule $T_{d-1}$ of $Z_{d-1}$ which is in $\mathcal{B}$, contains $Q_{d-1}$, maps to $T_{d}$ and surjects onto $K_{d}$. Proceeding inductively, we construct, for each $i=1,2, \ldots, d-2$, a submodule $T_{i}$ of $Z_{i}$ which is in $\mathcal{B}$, contains $Q_{i}$, maps 
to $T_{i+1}$ and surjects onto the kernel of the map from $T_{i+1}$ to $T_{i+2}$. This gives the commutative diagram

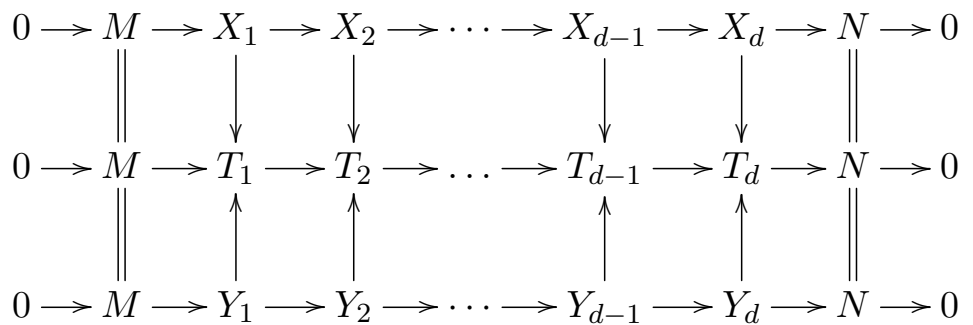

in $\mathcal{B}$ with exact rows. The above implies that the natural map

$$
\operatorname{Ext}_{\mathcal{B}}^{d}(N, M) \rightarrow \operatorname{Ext}_{\mathcal{A}}^{d}(N, M)
$$

is injective.

The construction above also says that for an arbitrary exact sequence

$$
0 \rightarrow M \rightarrow Z_{1} \longrightarrow Z_{2} \rightarrow \cdots \rightarrow Z_{d-1} \rightarrow Z_{d} \rightarrow N \rightarrow 0
$$

in $\mathcal{A}$ with $M, N \in \mathcal{B}$ there is a commutative diagram

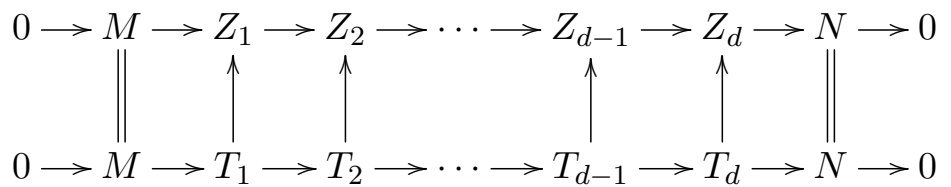

with exact rows and such that the second row is in $\mathcal{B}$. This means that the natural map (2) is surjective and hence bijective, completing the proof.

As an immediate corollary from Proposition 3 we obtain:

Corollary 1. For an associative algebra $A$ we have that

(i) A-fmod is extension full in A-lfmod,

(ii) A-mod is extension full in A-Mod provided that $A$ is Noetherian.

\subsection{Adjunction lemma}

The following statement is standard when dealing with categories with enough projective or injective objects. We failed to find it in the literature in the generality we need, so we provide a proof without the use of projective or injective objects.

Proposition 4 (adjunction lemma). Let $\mathcal{A}$ and $\mathcal{B}$ be two Abelian categories and (F, G) an adjoint pair of exact functors $\mathrm{F}: \mathcal{A} \rightarrow \mathcal{B}$ and $\mathrm{G}: \mathcal{B} \rightarrow \mathcal{A}$. Then for every $d \in \mathbb{Z}_{\geq 0}, N \in \mathcal{A}$ and $M \in \mathcal{B}$ there are isomorphisms

$$
\operatorname{Ext}_{\mathcal{B}}^{d}(\mathrm{~F}(N), M) \cong \operatorname{Ext}_{\mathcal{A}}^{d}(N, \mathrm{G}(M))
$$

natural in both $N$ and $M$.

Proof. Applying F to an exact sequence

$$
0 \longrightarrow \mathrm{G}(M) \longrightarrow X_{1} \longrightarrow X_{2} \longrightarrow \cdots \longrightarrow X_{d-1} \longrightarrow X_{d} \longrightarrow N \longrightarrow 0
$$


in $\mathcal{A}$, gives an exact sequence

$$
0 \rightarrow \mathrm{FG}(M) \rightarrow \mathrm{F}\left(X_{1}\right) \rightarrow \mathrm{F}\left(X_{2}\right) \rightarrow \cdots \rightarrow \mathrm{F}\left(X_{d-1}\right) \rightarrow \mathrm{F}\left(X_{d}\right) \rightarrow \mathrm{F}(N) \rightarrow 0
$$

in $\mathcal{B}$. Denote by $\mathrm{K}$ the kernel of the adjunction natural transformation $\mathrm{FG} \rightarrow \mathrm{Id}_{\mathcal{B}}$. Then we have the following commutative diagram

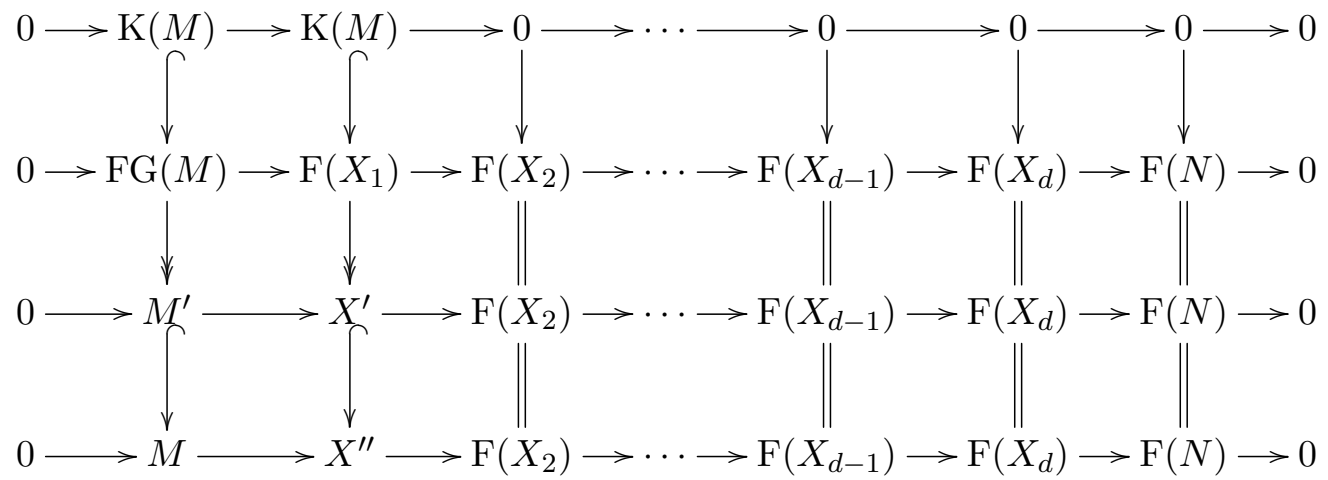

with exact rows. Here the homomorphism from the first to the second row is given by the natural embedding $\mathrm{K} \hookrightarrow \mathrm{FG}$ and the third row is just the corresponding cokernel with the morphism from the second to the third row being the canonical projection. In particular, $M^{\prime}:=\mathrm{FG}(M) / \mathrm{K}(M)$. The homomorphism from $M^{\prime}$ to $M$ is the natural inclusion (coming from the definition of $\mathrm{K}$ ) and, finally, $X^{\prime \prime}$ is defined as the push-out and the map to $\mathrm{F}\left(X_{2}\right)$ is given by the universal property of push-outs. Functoriality of the construction yields a group homomorphism

$$
\Phi: \operatorname{Ext}_{\mathcal{A}}^{d}(N, \mathrm{G}(M)) \rightarrow \operatorname{Ext}_{\mathcal{B}}^{d}(\mathrm{~F}(N), M) .
$$

Applying $\mathrm{G}$ to an exact sequence

$$
0 \longrightarrow M \longrightarrow Y_{1} \longrightarrow Y_{2} \longrightarrow \cdots \longrightarrow Y_{d-1} \longrightarrow Y_{d} \longrightarrow \mathrm{F}(N) \longrightarrow 0
$$

in $\mathcal{B}$, gives an exact sequence

$$
0 \rightarrow \mathrm{G}(M) \rightarrow \mathrm{G}\left(Y_{1}\right) \rightarrow \mathrm{G}\left(Y_{2}\right) \rightarrow \cdots \rightarrow \mathrm{G}\left(Y_{d-1}\right) \rightarrow \mathrm{G}\left(Y_{d}\right) \rightarrow \mathrm{GF}(N) \rightarrow 0
$$

in $\mathcal{A}$. Denote by $\mathrm{C}$ the cokernel of the adjunction natural transformation $\operatorname{Id}_{\mathcal{A}} \rightarrow$ GF. Then we have the following commutative diagram

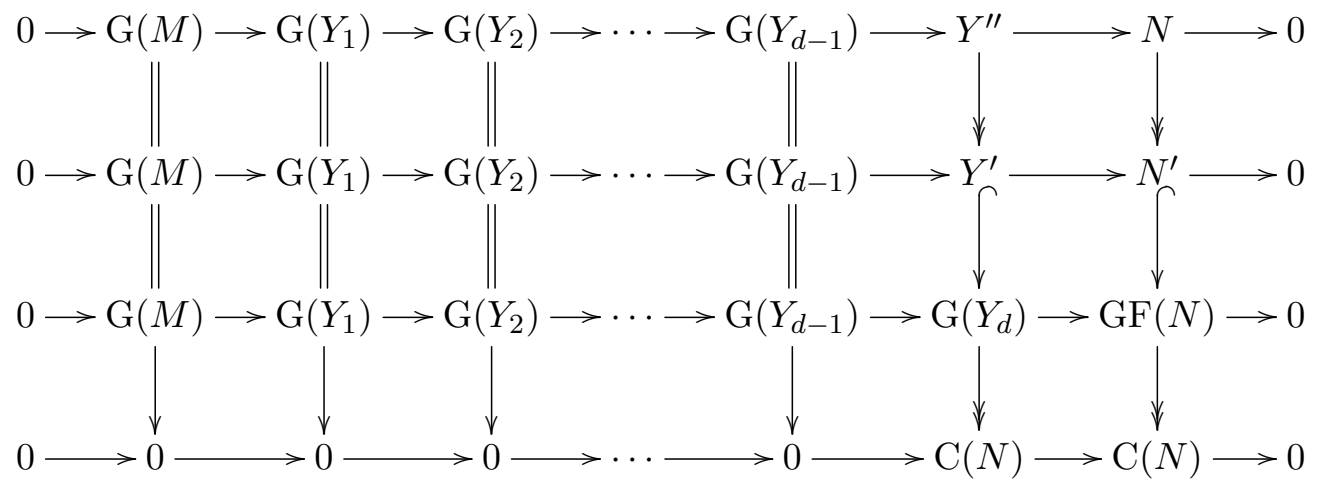

with exact rows. Here the homomorphism from the third to the last row is given by the natural projection of GF $\rightarrow \mathrm{C}$ and the second row is just the corresponding kernel with the morphism from the second to the third row being the canonical injection. In particular, $\mathrm{C}(N):=\operatorname{GF}(M) / N^{\prime}$. The homomorphism from $N$ to $N^{\prime}$ is the natural surjection and, finally, 
$Y^{\prime \prime}$ is defined as the pullback and the map from $\mathrm{G}\left(Y_{d-1}\right)$ is given by the universal property of pullbacks. Functoriality of the construction yields a group homomorphism

$$
\Psi: \operatorname{Ext}_{\mathcal{B}}^{d}(\mathrm{~F}(N), M) \rightarrow \operatorname{Ext}_{\mathcal{A}}^{d}(N, \mathrm{G}(M)) .
$$

Finally, we claim that $\Phi$ and $\Psi$ are inverses of each other. Consider the following commutative diagram:

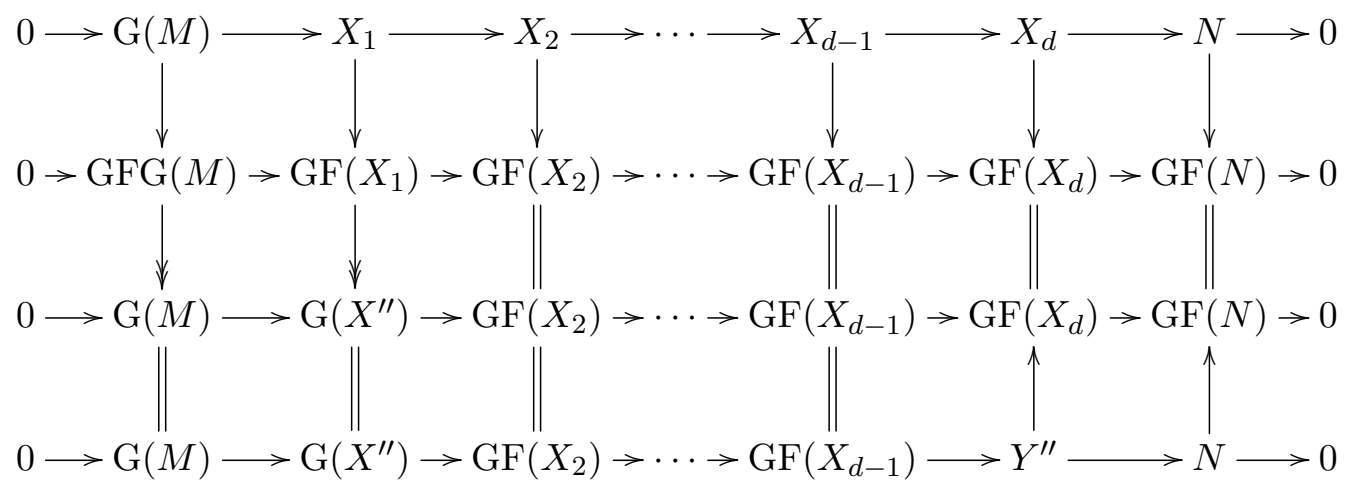

Here the second row is obtained from the first one by applying GF and the homomorphism from the first to the second row is given by adjunction $\operatorname{Id}_{\mathcal{A}} \rightarrow \mathrm{GF}$. The second and the third rows and the homomorphism between them are given by applying the exact functor $\mathrm{G}$ to the two middle rows of (3). Note that, by construction, application of $\mathrm{G}$ identifies the two last rows of (3), that is $\mathrm{G}\left(M^{\prime}\right) \cong \mathrm{G}(M)$ and $\mathrm{G}\left(X^{\prime}\right) \cong \mathrm{G}\left(X^{\prime \prime}\right)$ (this follows from surjectivity of the natural transformation GFG $\rightarrow \mathrm{G}$ given by adjunction). Consequently, from the adjunction identities we have that the composition of the maps in the first column is the identity on $\mathrm{G}(M)$. Finally, the last row and the homomorphism to the third row are given by the definition of $\Psi$. In particular, from the construction we have that the image of $N$ in the second row (coming from the first row) and in the third row (coming from the last row) coincide. Hence diagram (4) gives rise to a diagram

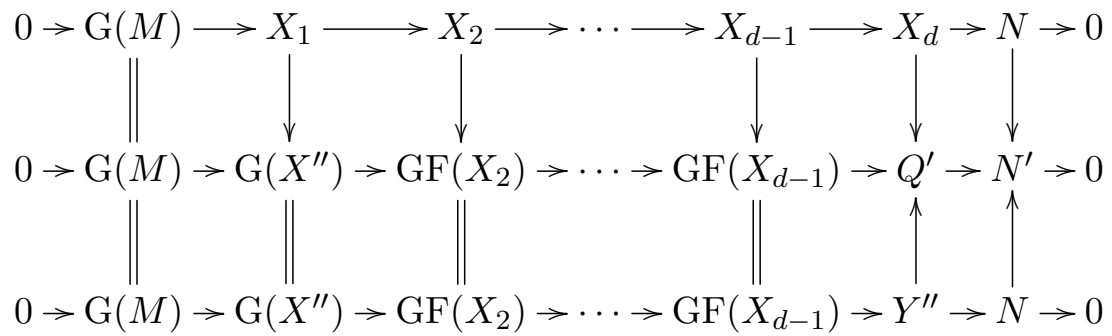

with exact rows, where $N^{\prime}$ is the image of $N$ in $\operatorname{GF}(N)$ and $Q^{\prime}$ is the full preimage of $N^{\prime}$. Pulling back along the epimorphism $N \rightarrow N^{\prime}$ gives a commutative diagram

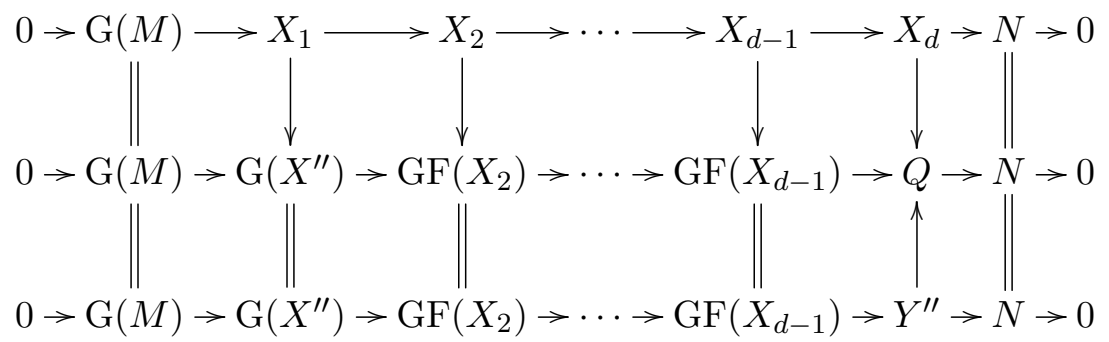

with exact rows. The last diagram shows that the extensions given by the first and the last rows coincide, which proves that $\Psi \Phi$ is the identity map.

The claim that $\Phi \Psi$ is the identity map is proved similarly. This completes the proof. 


\section{Gelfand-Zeitlin modules}

Notation. For a Lie algebra $\mathfrak{a}$ we denote by $U(\mathfrak{a})$ its universal enveloping algebra and by $Z(\mathfrak{a})$ the center of $U(\mathfrak{a})$.

\subsection{Gelfand-Zeitlin subalgebra of $\mathfrak{g l}_{n}$}

For $k \in \mathbb{Z}_{>0}$ denote by $\mathfrak{g}_{k}$ the Lie algebra $\mathfrak{g l}_{k}(\mathbb{C})$. Set $U_{k}=U\left(\mathfrak{g}_{k}\right)$ and $Z_{k}=Z\left(\mathfrak{g}_{k}\right)$. We consider the usual chain

$$
\mathfrak{g}_{1} \subset \mathfrak{g}_{2} \subset \mathfrak{g}_{3} \subset \cdots \subset \mathfrak{g}_{n}
$$

of "left upper corner" embeddings of Lie algebras as depicted on the following picture:

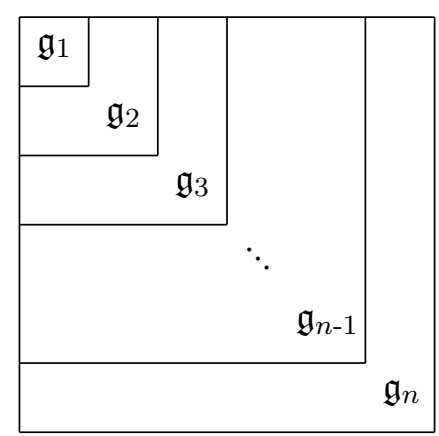

This gives rise to the chain

$$
U_{1} \subset U_{2} \subset U_{3} \subset \cdots \subset U_{n}
$$

of embeddings of associative algebras. The subalgebra $\Gamma$ of $U:=U_{n}$ generated by all centers $Z_{k}$, where $k=1,2, \ldots, n$, is called the Gelfand-Zeitlin subalgebra. We set $\mathfrak{g}:=\mathfrak{g}_{n}$.

The algebra $\Gamma$ is obviously commutative, moreover, $\Gamma$ is a polynomial algebra in $\frac{n(n+1)}{2}$ variables (these can be taken to be generators of $Z_{k}$ for $k=1,2, \ldots, n$ ), see [23] or [50, Chapter X]. Considered as a subalgebra of $U, \Gamma$ is a Harish-Chandra subalgebra in the sense of [12, Section 1 ], which means that every finitely generated $\Gamma$-subbimodule of $U$ is already finitely generated both as a left and as a right $\Gamma$-module, see [12, Theorem 24]. Furthermore, $U$ is free both as a left and as a right $\Gamma$-module, see [44]. Consequently, the usual induction and coinduction functors

$$
\operatorname{Ind}_{\Gamma}^{U}:=U \bigotimes_{\Gamma} \quad \text { and } \quad \operatorname{Coind}_{\Gamma}^{U}:=\operatorname{Hom}_{\Gamma}\left(U,_{-}\right)
$$

are exact.

\subsection{Gelfand-Zeitlin modules}

The category $\mathscr{G} \mathscr{Z}$ of Gelfand-Zeitlin-modules for $U$ is defined as the full subcategory of $U$-mod (the category of finitely generated $U$-modules), which consists of those $M \in U$-mod on which the action of $\Gamma$ is locally finite, that is $\operatorname{dim}(\Gamma v)<\infty$ for all $v \in M$. The category $\mathscr{G} \mathscr{Z}$ is a Serre subcategory of $U$-mod as $U$ is Noetherian.

Write $\operatorname{Specm}(\Gamma)$ for the set of maximal ideals in $\Gamma$. As $\Gamma$ is a polynomial algebra, we have the decomposition

$$
\Gamma-\mathrm{fmod}=\bigoplus_{\mathbf{m} \in \operatorname{Specm}(\Gamma)} \Gamma-\mathrm{fmod}_{\mathbf{m}}
$$


where $\Gamma$-fmod $\mathbf{m}_{\mathbf{m}}$ denotes the full subcategory of $\Gamma$-fmod consisting of all objects annihilated by some power of $\mathbf{m}$. From this decomposition, consider the functor

$$
\operatorname{Ind}_{\Gamma, \mathbf{m}}^{U}: \Gamma-\operatorname{fmod}_{\mathbf{m}} \rightarrow \mathscr{G} \mathscr{Z}
$$

defined as the restriction of $\operatorname{Ind}_{\Gamma}^{U}$ to $\Gamma-\mathrm{fmod}_{\mathbf{m}}$.

Note that modules in $\mathscr{G} \mathscr{Z}$ are usually infinite-dimensional and hence the usual restriction $\operatorname{Res}_{\Gamma}^{U}$ ends up in $\Gamma$-lfmod and not in $\Gamma$-fmod. However, from [21, Corollary 5.3(a)] it follows that for any $M \in \mathscr{G} \mathscr{Z}$ and $\mathbf{m} \in \operatorname{Specm}(\Gamma)$ the space

$$
M_{\mathbf{m}}:=\left\{v \in \operatorname{Res}_{\Gamma}^{U} M: \mathbf{m}^{i} v=0 \text { for some } i\right\}
$$

is finite-dimensional. This allows us to define the functor

$$
\operatorname{Res}_{\Gamma, \mathbf{m}}^{U}: \mathscr{G} \mathscr{Z} \rightarrow \Gamma-\mathrm{fmod}_{\mathbf{m}},
$$

which sends $M$ to $M_{\mathbf{m}}$ and is defined on morphisms by restriction. Then the usual adjunction between induction and restriction implies that $\operatorname{Ind}_{\Gamma, \mathbf{m}}^{U}$ is left adjoint to $\operatorname{Res}_{\Gamma, \mathbf{m}}^{U}$. The functor $\operatorname{Res}_{\Gamma, \mathbf{m}}^{U}$ is, in turn, left adjoint to the restriction

$$
\operatorname{Coind}_{\Gamma, \mathbf{m}}^{U}: \Gamma-\operatorname{fmod}_{\mathbf{m}} \rightarrow \mathscr{G} \mathscr{Z}
$$

of the coinduction functor to $\Gamma-\mathrm{fmod}_{\mathbf{m}}$.

\subsection{The main result}

Our main result in this section is the following:

Theorem 1. The category $\mathscr{G} \mathscr{Z}$ is extension full in $U$-Mod.

Proof. To prove Theorem 1, we would like to apply Proposition 1 for $\mathcal{A}=U$-Mod, $\mathcal{B}=$ $\mathscr{G} \mathscr{Z}$ and $\mathcal{B}_{0}$ being the full subcategory of $\mathcal{B}$ consisting of all $U$-modules isomorphic to $\operatorname{Ind}_{\Gamma}^{U} N$ for a finite-dimensional $\Gamma$-module $N$. Taking the assumptions of Proposition 1 into account, Theorem 1 reduces to the following lemma:

Lemma 1. Let $Q \in \mathscr{G} \mathscr{Z}, N$ be a finite-dimensional $\Gamma$-module and $M=\operatorname{Ind}_{\Gamma}^{U} N$. Then $\iota_{M, Q}^{d}$ is an isomorphism for every $d \in \mathbb{Z}_{\geq 0}$.

Proof. By additivity, we may assume $N \in \Gamma-\operatorname{fmod}_{\mathbf{m}}$ for some $\mathbf{m} \in \operatorname{Specm}(\Gamma)$. The image of the functor $\operatorname{Ind}_{\Gamma, \mathbf{m}}^{U}: \Gamma-\operatorname{fmod}_{\mathbf{m}} \rightarrow \mathcal{A}$ belongs to $\mathcal{B}$. We show that for every $d \in \mathbb{Z}_{\geq 0}$, any $N \in \Gamma$ - fmod $_{\mathbf{m}}$ and any $Q \in \mathscr{G} \mathscr{Z}$ we have the isomorphisms

$$
\begin{aligned}
& \operatorname{Ext}_{\mathcal{A}}^{d}\left(\operatorname{Ind}_{\Gamma, \mathbf{m}}^{U} N, Q\right) \cong \operatorname{Ext}_{\Gamma-\operatorname{Mod}^{\prime}}^{d}\left(N, \operatorname{Res}_{\Gamma}^{U} Q\right), \\
& \operatorname{Ext}_{\Gamma-\operatorname{Mod}}^{d}\left(N, \operatorname{Res}_{\Gamma}^{U} Q\right) \cong \operatorname{Ext}_{\Gamma-\bmod _{\mathbf{m}}}^{d}\left(N, \operatorname{Res}_{\Gamma, \mathbf{m}}^{U} Q\right), \\
& \operatorname{Ext}_{\Gamma-\bmod _{\mathbf{m}}}^{d}\left(N, \operatorname{Res}_{\Gamma, \mathbf{m}}^{U} Q\right) \cong \operatorname{Ext}_{\mathcal{B}}^{d}\left(\operatorname{Ind}_{\Gamma, \mathbf{m}}^{U} N, Q\right) .
\end{aligned}
$$

Since, by construction, these three isomorphisms together with the morphism $\iota_{M, Q}^{d}$ will yield a commutative square, we get that $\iota_{M, Q}^{d}$ is an isomorphism.

Isomorphism (6) follows from [7, Lemma 17]. Isomorphisms (5) and (7) follow from adjunction lemma (Proposition 4). Note that adjunction lemma applies here since $U$ is free over $\Gamma$.

Theorem 1 now follows. 


\subsection{The global dimension of $\mathscr{G} \mathscr{Z}$}

Corollary 2. We have gl.dim $(\mathscr{G} \mathscr{Z})=\operatorname{gl} \cdot \operatorname{dim}(U-\mathrm{Mod})=\operatorname{dim} \mathfrak{g}$.

Proof. It is well-known, see e.g. [49, Corollary 7.7.3], that the trivial $\mathfrak{g}$-module $\mathbb{C}$ has maximal possible projective dimension in $U$-Mod, namely dim $\mathfrak{g}$. Since we obviously have $\mathbb{C} \in \mathscr{G} \mathscr{Z}$, the claim follows from Theorem 1.

Remark 1. The category $\mathscr{G} \mathscr{Z}$ decomposes into a direct sum of indecomposable blocks, see [12, Theorem 24]. The proof of Corollary 2 implies that the block containing the trivial $\mathfrak{g}$-module has global dimension $\operatorname{dim} \mathfrak{g}=n^{2}$. However, most of the blocks in $\mathscr{G} \mathscr{Z}$, namely all the socalled strongly generic blocks in the sense of [36, Section 3], are equivalent to the category of finite dimension modules over $\Gamma_{\mathbf{m}}$, the completion of $\Gamma$ with respect to a maximal ideal $\mathbf{m}$ (this equivalence is induced by $\left.\operatorname{Ind}_{\Gamma, \mathbf{m}}^{U}\right)$, and hence have global dimension $\frac{n(n+1)}{2}$, that is the Krull dimension of $\Gamma$.

\section{Whittaker modules}

\subsection{The category of Whittaker modules}

Let $\mathfrak{g}$ be a semi-simple finite-dimensional complex Lie algebra with a fixed triangular decomposition $\mathfrak{g}=\mathfrak{n}_{-} \oplus \mathfrak{h} \oplus \mathfrak{n}_{+}$. Consider the subalgebra $R=Z(\mathfrak{g}) U\left(\mathfrak{n}_{+}\right)$in $U(\mathfrak{g})$. Note that $R$ is not commutative unless $\mathfrak{g}$ is a direct sum of copies of $\mathfrak{s l}_{2}$. Set $U=U(\mathfrak{g})$.

Denote by $\mathscr{W}$ the full subcategory of $U$-mod (the category of finitely generated $U$-modules) consisting of all $\mathfrak{g}$-modules which are locally finite with respect to the action of $R$ (cf. [38, Definition 1.5]). Objects in $\mathscr{W}$ will be called Whittaker modules, which is a slight modification of the original notion from [29].

\subsection{Simple finite-dimensional $R$-modules}

In order to better understand the category $\mathscr{W}$, we start with a classification of simple finitedimensional $R$-modules. For this we would need the following fact.

Proposition 5. The algebra $R$ is isomorphic to $Z(\mathfrak{g}) \otimes_{\mathbb{C}} U\left(\mathfrak{n}_{+}\right)$.

Proof. From the PBW Theorem it follows that the multiplication map

$$
U(\mathfrak{h}) \otimes_{\mathbb{C}} U\left(\mathfrak{n}_{+}\right) \rightarrow U(\mathfrak{h}) U\left(\mathfrak{n}_{+}\right)
$$

is bijective. Injectivity of the Harish-Chandra homomorphism $Z(\mathfrak{g}) \rightarrow U(\mathfrak{h})$ yields that the $U(\mathfrak{h})$ components of different elements in $Z(\mathfrak{g})$ are different. Hence (8) implies that the surjective homomorphism $Z(\mathfrak{g}) \otimes_{\mathbb{C}} U\left(\mathfrak{n}_{+}\right) \rightarrow R$ given by multiplication is, in fact, injective, and hence an isomorphism (see also [29, Section 3.3]).

Fix a maximal ideal $\mathbf{m}$ in $Z(\mathfrak{g})$ and a linear map $\chi: \mathfrak{n}_{+} /\left[\mathfrak{n}_{+}, \mathfrak{n}_{+}\right] \rightarrow \mathbb{C}$ and denote by $V_{\mathbf{m}, \chi}$ the space $\mathbb{C}$ endowed with the action of $Z(\mathfrak{g})$ via the projection $Z(\mathfrak{g}) / \mathbf{m} \cong \mathbb{C}$ and with the action of $U\left(\mathfrak{n}_{+}\right)$via $\chi$. The following statement shows that this gives a complete and irredundant list of pairwise non-isomorphic simple finite-dimensional $R$-modules.

\section{Proposition 6.}

(i) For each $\mathbf{m}$ and $\chi$ as above, $V_{\mathbf{m}, \chi}$ is a simple $R$-module.

(ii) Each simple finite-dimensional $R$-module is isomorphic to $V_{\mathbf{m}, \chi}$ for some $\mathbf{m}$ and $\chi$ as above.

(iii) We have $V_{\mathbf{m}, \chi} \cong V_{\mathbf{m}^{\prime}, \chi^{\prime}}$ if and only if $\mathbf{m}=\mathbf{m}^{\prime}$ and $\chi=\chi^{\prime}$. 
Proof. We have that $V_{\mathbf{m}, \chi}$ is a $Z(\mathfrak{g}) \otimes_{\mathbb{C}} U\left(\mathfrak{n}_{+}\right)$-module by construction. Hence claim (i) follows from Proposition 5. Claim (iii) is clear by construction.

To prove claim (ii), we note that $R$ is a finitely generated complex algebra and hence every simple $R$-module admits a central character by Dixmier's version of Schur's lemma, see [9, Proposition 2.6.8]. Therefore, from Proposition 5 it follows that simple finite-dimensional $R$ modules are exactly simple finite-dimensional $U\left(\mathfrak{n}_{+}\right)$-modules with the action of $Z(\mathfrak{g})$ given via the projection $Z(\mathfrak{g}) / \mathbf{m} \cong \mathbb{C}$ for some $\mathbf{m}$. Since $\mathfrak{n}_{+}$is nilpotent, all simple finite-dimensional $\mathfrak{n}_{+}$ have dimension one by Lie's theorem, see [9, Corollary 1.3.13]. The claim follows.

\subsection{The categories $R$-fmod and $R$-lfmod}

For a maximal ideal $\mathrm{m}$ in $Z(\mathfrak{g})$ and a linear map $\chi: \mathfrak{n}_{+} /\left[\mathfrak{n}_{+}, \mathfrak{n}_{+}\right] \rightarrow \mathbb{C}$ denote by $R$-fmod $\mathbf{m}_{\mathbf{m}, \chi}$ the full subcategory of $R$-fmod consisting of all modules for which all simple composition subquotients are isomorphic to $V_{\mathbf{m}, \chi}$. Define similarly the subcategory $R$-lfmod $\mathbf{m}, \chi_{\text {of }} R$-lfmod.

Proposition 7. We have decompositions

$$
R \text {-fmod } \cong \bigoplus_{\mathbf{m}, \chi} R-\operatorname{fmod}_{\mathbf{m}, \chi} \quad \text { and } \quad R \text {-lfmod } \cong \bigoplus_{\mathbf{m}, \chi} R-\text { lfmod }_{\mathbf{m}, \chi}
$$

Proof. To prove the claim it is sufficient to check that

$$
\operatorname{Ext}_{R}^{1}\left(V_{\mathbf{m}, \chi}, V_{\mathbf{m}^{\prime}, \chi^{\prime}}\right)=0
$$

unless $\mathbf{m}=\mathbf{m}^{\prime}$ and $\chi=\chi^{\prime}$.

If $\mathbf{m} \neq \mathbf{m}^{\prime}$, then (9) is clear as $Z(\mathfrak{g})$ is central in $R$. Assume $\chi \neq \chi^{\prime}$ and consider some short exact sequence

$$
0 \rightarrow V_{\mathbf{m}, \chi^{\prime}} \rightarrow M \rightarrow V_{\mathbf{m}, \chi} \rightarrow 0 .
$$

As $\chi \neq \chi^{\prime}$, there is $a \in \mathfrak{n}_{+}$whose action on $M$ has two different eigenvalues $\chi(a) \neq \chi^{\prime}(a)$. Let $v$ and $w$ be the corresponding non-zero eigenvectors in $M$ (note that each of them is unique up to a non-zero scalar). Then $\mathbb{C} w$ coincides with the image of $V_{\mathbf{m}, \chi^{\prime}}$ in $M$. We claim that $\mathbb{C} v$ is an $R$-submodule (which would mean that the short exact sequence (10) splits thus completing the proof).

Indeed, we have $Z(\mathfrak{g}) \mathbb{C} v \subset \mathbb{C} v$ as $\mathbb{C} v=\{x \in M: a x=\chi(a) x\}$ and $Z(\mathfrak{g})$ commutes with $a$. Consider some filtration

$$
0=X_{0} \subset X_{1} \subset \cdots \subset X_{k}=\mathfrak{n}_{+}
$$

such that $\left[a, X_{i}\right] \subset X_{i-1}$ for all $i$. Such filtration exists as $\mathfrak{n}_{+}$is nilpotent. The elements of $X_{1}$ commute with $a$ and hence $X_{1} \mathbb{C} v \subset \mathbb{C} v$. Let $b \in X_{2}$ and assume that $b v=\alpha v+\beta w$. Then, on the one hand,

$$
a b v=\chi(a)(\alpha v+\beta w)+\left(\chi^{\prime}(a)-\chi(a)\right) \beta w=\chi(a) b v+\left(\chi^{\prime}(a)-\chi(a)\right) \beta w .
$$

On the other hand, $a b v=\chi(a) b v+[a, b] v$. As $[a, b] \in X_{1}, X_{1} v \subset \mathbb{C} v$ and $v$ and $w$ are linearly independent, we get $[a, b] v=\left(\chi^{\prime}(a)-\chi(a)\right) \beta w=0$, that is $\beta=0$. Therefore $b v \in \mathbb{C} v$ and thus $X_{2} v \subset \mathbb{C} v$. Proceeding inductively, we get $\mathfrak{n}_{+} v \subset \mathbb{C} v$ and the proof is complete.

Note that the algebra $R$ is isomorphic to the enveloping algebra of the nilpotent Lie algebra $\mathfrak{n}_{+} \oplus \mathfrak{a}$, where $\mathfrak{a}$ is an Abelian Lie algebra of dimension $\operatorname{dim}(\mathfrak{h})$, see Proposition 5. In particular $R \cong U(\mathfrak{n})$ for some solvable Lie algebra $\mathfrak{n}$. Therefore we will be able to make use of the following result taken from [8, Proposition 1], see also [10] and [16]. 
Lemma 2. Let $\mathfrak{n}$ be a solvable Lie algebra. Any module $V \in U(\mathfrak{n})$-lfmod has an injective hull $I_{V}$ in $U(\mathfrak{n})$-Mod, moreover, $I_{V} \in U(\mathfrak{n})$-lfmod.

We note that existence of injective hulls in $U(\mathfrak{n})$-Mod is due, in much bigger generality, to Baer [1], see also [15]. Lemma 2 implies the following.

Proposition 8. Let $\mathfrak{n}$ be a finite-dimensional solvable Lie algebra. Then the category $U(\mathfrak{n})$-lfmod has enough injective objects and, moreover, $U(\mathfrak{n})$-lfmod is extension full in $U(\mathfrak{n})$-Mod.

Proof. As $U(\mathfrak{n})$-lfmod is a Serre subcategory of $U(\mathfrak{n})$-Mod, the injective hulls in $U(\mathfrak{n})$-Mod of Lemma 2 are automatically injective hulls in $U(\mathfrak{n})$-lfmod. This proves that $U(\mathfrak{n})$-lfmod has enough injective objects.

As $U(\mathfrak{n})$-lfmod is a locally Noetherian Grothendieck category (see [30, Appendix A] or [47]), it follows that each injective object in this category is a coproduct of indecomposable injective objects and this decomposition is unique up to isomorphism. Since $\mathfrak{n}$ is finite-dimensional, $U(\mathfrak{n})$ is Noetherian and hence a coproduct of injective objects in $U(\mathfrak{n})$-Mod is injective, see [32, Proposition 1.2]. This implies that any injective object in $U(\mathfrak{n})$-lfmod is also injective when regarded as a module in $U(\mathfrak{n})$-Mod. The extension fullness thus follows from Proposition 2.

As a consequence, we obtain the following statement.

Corollary 3. The category $R$-lfmod is extension full in $R$-Mod.

\section{$4.4 R$ versus $U(\mathfrak{g})$}

Proposition 9. The algebra $U(\mathfrak{g})$ is free both as a left and as a right $R$-module.

Proof. We prove the statement for the right module structure. The claim for the left module structure then follows by applying the canonical antiautomorphism of $U(\mathfrak{g})$ generated by $x \mapsto-x$ for $x \in \mathfrak{g}$.

Choose a basis $Y_{1}, Y_{2}, \ldots, Y_{k}$ in $\mathfrak{n}_{-}$, a basis $H_{1}, H_{2}, \ldots, H_{l}$ in $\mathfrak{h}$ and a basis $X_{1}, X_{2}, \ldots, X_{k}$ in $\mathfrak{n}_{+}$. Then

$$
\left\{Y_{1}^{s_{1}} Y_{2}^{s_{2}} \cdots Y_{k}^{s_{k}} H_{1}^{t_{1}} H_{2}^{t_{2}} \cdots H_{l}^{t_{l}} X_{1}^{r_{1}} X_{2}^{r_{2}} \cdots X_{k}^{r_{k}}: s_{i}, t_{i}, r_{i} \in \mathbb{Z}_{\geq 0}\right\}
$$

is a basis in $U(\mathfrak{g})$ by the PBW theorem. For an element $v$ in the above basis, the degree of of $v$ is the degree of its $\mathfrak{n}_{+}$-component $X_{1}^{r_{1}} X_{2}^{r_{2}} \cdots X_{k}^{r_{k}}$, where the grading on $U\left(\mathfrak{n}_{+}\right)$is defined by giving constants degree 0 and simple root vectors degree 1 . This induces an $\mathbb{Z}_{\geq 0}$-grading on the vector space $U(\mathfrak{g})$.

Choose a basis $f_{1}, f_{2}, \ldots, f_{m}$ of $U(\mathfrak{h})$ as the free module over the algebra of invariants in $U(\mathfrak{h})$ with respect to the dot-action of the Weyl group $W$ of $\mathfrak{g}$. This algebra of invariants is exactly the image of $Z(\mathfrak{g})$ under the Harish-Chandra isomorphism, moreover, $m=|W|$. We fix free generators of this algebra of invariants as $p_{1}, p_{2}, \ldots, p_{l}$. Then we choose free generators $z_{1}, z_{2}, \ldots, z_{l}$ of $Z(\mathfrak{g})$ as a polynomial algebra by requiring $z_{i}-p_{i} \in U(\mathfrak{g}) \mathfrak{n}_{+}$. This gives that the set

$$
\underline{\mathbf{B}}:=\left\{Y_{1}^{s_{1}} Y_{2}^{s_{2}} \cdots Y_{k}^{s_{k}} f_{h} p_{1}^{t_{1}} p_{2}^{t_{2}} \cdots p_{l}^{t_{l}} X_{1}^{r_{1}} X_{2}^{r_{2}} \cdots X_{k}^{r_{k}}: s_{i}, t_{i}, r_{i} \in \mathbb{Z}_{\geq 0}, h=1, \ldots, m\right\}
$$

is a basis of $U(\mathfrak{g})$.

Consider the set

$$
\mathbf{B}:=\left\{Y_{1}^{s_{1}} Y_{2}^{s_{2}} \cdots Y_{k}^{s_{k}} f_{j}: s_{i} \in \mathbb{Z}_{\geq 0}, j=1,2, \ldots, m\right\} .
$$

By Proposition 5, the set

$$
\mathbf{B}^{\prime}:=\left\{z_{1}^{q_{1}} z_{2}^{q_{2}} \cdots z_{l}^{q_{l}} X_{1}^{r_{1}} X_{2}^{r_{2}} \cdots X_{k}^{r_{k}}: q_{i}, r_{i} \in \mathbb{Z}_{\geq 0}\right\}
$$


is a basis of $R$. Multiplying $Y_{1}^{s_{1}} Y_{2}^{s_{2}} \cdots Y_{k}^{s_{k}} f_{j}$ on the right with $z_{1}^{q_{1}} z_{2}^{q_{2}} \cdots z_{l}^{q_{l}} X_{1}^{r_{1}} X_{2}^{r_{2}} \cdots X_{k}^{r_{k}}$ gives

$$
Y_{1}^{s_{1}} Y_{2}^{s_{2}} \cdots Y_{k}^{s_{k}} f_{j} z_{1}^{q_{1}} z_{2}^{q_{2}} \cdots z_{l}^{q_{l}} X_{1}^{r_{1}} X_{2}^{r_{2}} \cdots X_{k}^{r_{k}} .
$$

This can be written as the sum of

$$
Y_{1}^{s_{1}} Y_{2}^{s_{2}} \cdots Y_{k}^{s_{k}} f_{j} p_{1}^{q_{1}} p_{2}^{q_{2}} \cdots p_{l}^{q_{l}} X_{1}^{r_{1}} X_{2}^{r_{2}} \cdots X_{k}^{r_{k}}
$$

plus a linear combination of elements $v$ from the basis (11) that have strictly higher degree than the degree of the homogeneous element (12). Consider the multiplication map

$$
\operatorname{Span}(\mathbf{B}) \otimes_{\mathbb{C}} R \rightarrow U(\mathfrak{g}) .
$$

Using the induction on the total degree of the Cartan part, it is easy to check that this map is surjective. At the same time, we have the induced map

$$
\varphi: \mathbf{B} \times \mathbf{B}^{\prime} \rightarrow U(\mathfrak{g}) .
$$

For $x \in \mathbf{B}$ and $y \in \mathbf{B}^{\prime}$, by taking the unique element from (11) of minimal degree which appears in the expression of $\varphi(x, y)$ with a non-zero coefficient, induces a bijection from $\mathbf{B} \times \mathbf{B}^{\prime}$ to $\underline{\mathbf{B}}$. This implies that all elements in $\mathbf{B B}^{\prime}$ are linearly independent and hence $\mathbf{B}$ is a basis of $U(\mathfrak{g})$ as a free right $R$-module.

Proposition 10. For every finite-dimensional $R$-module $V$ the induced $\mathfrak{g}$-module $M(V):=$ $\operatorname{Ind}_{R}^{U(\mathfrak{g})} V$ has finite length.

Proof. It is enough to prove the claim for $V=V_{\mathbf{m}, \chi}$, where $\mathbf{m}$ is a maximal ideal in $Z(\mathfrak{g})$ and $\chi: \mathfrak{n}_{+} /\left[\mathfrak{n}_{+}, \mathfrak{n}_{+}\right] \rightarrow \mathbb{C}$. In this case the statement follows from [38, Theorem 2.8].

Corollary 4. Every object in $\mathscr{W}$ has finite length.

Proof. Each $M \in \mathscr{W}$ is generated, as a $\mathfrak{g}$-module, by some finite-dimensional $R$-submodule $V$. By adjunction, $M$ is thus a quotient of $M(V)$ and hence the claim follows from Proposition 10 .

\subsection{The main result}

Our main result in this section is the following:

Theorem 2. The category $\mathscr{W}$ is extension full in $U$-Mod.

Proof. We start by proving extension fullness of the category $\widehat{\mathscr{W}}$ which is defined as the full subcategory of $U$-Mod consisting of all modules which are locally $R$-finite. The difference between $\widehat{\mathscr{W}}$ and $\mathscr{W}$ is that we drop the condition of being finitely generated.

We apply Proposition 1 for $\mathcal{A}=U$-Mod, $\mathcal{B}=\widehat{\mathscr{W}}$ and $\mathcal{B}_{0}$ being the full subcategory of $\mathcal{B}$ consisting of all $U$-modules isomorphic to $M(V)$ for some $V \in R$-lfmod.

Lemma 3. Let $Q \in \widehat{\mathscr{W}}$ and $V \in R$-lfmod. Then $\iota_{M(V), Q}^{d}$ is an isomorphism for every $d \in \mathbb{Z}_{\geq 0}$.

Proof. The image of the functor

$$
\operatorname{Ind}_{R}^{U}: R \text {-lfmod } \rightarrow \mathcal{A}
$$

belongs to $\mathcal{B}$ while the image of the functor

$$
\operatorname{Res}_{R}^{U}: \mathcal{B} \rightarrow R-\operatorname{Mod}
$$


belongs to $R$-lfmod. Therefore, to prove our lemma, it is enough to show that for every $d \in \mathbb{Z}_{\geq 0}$, any $N \in R$-lfmod and any $Q \in \mathscr{W}$ we have the isomorphisms

$$
\begin{aligned}
& \operatorname{Ext}_{\mathcal{A}}^{d}\left(\operatorname{Ind}_{R}^{U} N, Q\right) \cong \operatorname{Ext}_{R-\operatorname{Mod}}^{d}\left(N, \operatorname{Res}_{R}^{U} Q\right), \\
& \operatorname{Ext}_{R \text {-Mod }}^{d}\left(N, \operatorname{Res}_{R}^{U} Q\right) \cong \operatorname{Ext}_{R-\operatorname{lfmod}}^{d}\left(N, \operatorname{Res}_{R}^{U} Q\right), \\
& \operatorname{Ext}_{R \text {-lfmod }}^{d}\left(N, \operatorname{Res}_{R}^{U} Q\right) \cong \operatorname{Ext}_{\mathcal{B}}^{d}\left(\operatorname{Ind}_{R}^{U} N, Q\right) .
\end{aligned}
$$

Isomorphisms (13) and (15) follow from adjunction lemma (Proposition 4) which applies thanks to Proposition 9, while isomorphism (14) is Proposition 3. The claim follows.

Lemma 3 and Proposition 1 thus imply that $\widehat{\mathscr{W}}$ is extension full in $U$-Mod. Hence, to complete the proof of Theorem 2, it remains to note that, by Proposition 3 , the category $\mathscr{W}$ is extension full in $\widehat{\mathscr{W}}$.

\subsection{The global dimension of $\mathscr{W}$}

Corollary 5. We have gl.dim $(\mathscr{W})=\operatorname{gl} \cdot \operatorname{dim}(U-\operatorname{Mod})=\operatorname{dim} \mathfrak{g}$.

Proof. Mutatis mutandis Corollary 2.

Remark 2. The category $\mathscr{W}$ has a decomposition

$$
\mathscr{W} \cong \bigoplus_{\mathbf{m}, \chi} \mathscr{W}_{\mathbf{m}, \chi}
$$

where $\mathscr{W}_{\mathbf{m}, \chi}$ is the full subcategory consisting of all modules which restrict to $R-\operatorname{lfmod}_{\mathbf{m}, \chi}$, see [3, Theorem 9]. Corollary 5 says that one of these blocks, namely the one corresponding to the trivial central character and trivial $\chi$ has global dimension dim $\mathfrak{g}$. This particular block contains many simple objects. Most of the blocks contain only one simple object and are expected to have smaller global dimension. Note also that thick category $\mathcal{O}$ is a Serre subcategory of $\mathscr{W}$.

\section{Acknowledgements}

KC is a Postdoctoral Fellow of the Research Foundation - Flanders (FWO). VM is partially supported by the Swedish Research Council. A substantial part of this work was done during the visit of the authors to the CRM Thematic Semester "New Directions in Lie Theory" in Montreal. We thank CRM for hospitality and partial support. We thank the referees for helpful comments.

\section{References}

[1] Baer R., Abelian groups that are direct summands of every containing abelian group, Bull. Amer. Math. Soc. 46 (1940), 800-806.

[2] Bagci I., Christodoulopoulou K., Wiesner E., Whittaker categories and Whittaker modules for Lie superalgebras, Comm. Algebra 42 (2014), 4932-4947, arXiv:1201.5350.

[3] Batra P., Mazorchuk V., Blocks and modules for Whittaker pairs, J. Pure Appl. Algebra 215 (2011), 15521568, arXiv:0910.3540.

[4] Benkart G., Ondrus M., Whittaker modules for generalized Weyl algebras, Represent. Theory 13 (2009), 141-164, arXiv:0803.3570.

[5] Brüstle Th., König S., Mazorchuk V., The coinvariant algebra and representation types of blocks of category $\mathcal{O}$, Bull. London Math. Soc. 33 (2001), 669-681.

[6] Christodoulopoulou K., Whittaker modules for Heisenberg algebras and imaginary Whittaker modules for affine Lie algebras, J. Algebra 320 (2008), 2871-2890. 
[7] Coulembier K., Mazorchuk V., Some homological properties of the category O. III, arXiv:1404.3401.

[8] Dahlberg R.P., Injective hulls of Lie modules, J. Algebra 87 (1984), 458-471.

[9] Dixmier J., Enveloping algebras, Graduate Studies in Mathematics, Vol. 11, Amer. Math. Soc., Providence, RI, 1996.

[10] Donkin S., On the Hopf algebra dual of an enveloping algebra, Math. Proc. Cambridge Philos. Soc. 91 (1982), 215-224.

[11] Drozd Yu.A., Representations of Lie algebras $\mathfrak{s l}(2)$, Vīsnik Kï̌v. Unīv. Ser. Mat. Mekh. (1983), no. 25, 70-77.

[12] Drozd Yu.A., Futorny V.M., Ovsienko S.A., Harish-Chandra subalgebras and Gel'fand-Zetlin modules, in Finite-Dimensional Algebras and Related Topics (Ottawa, ON, 1992), NATO Adv. Sci. Inst. Ser. C Math. Phys. Sci., Vol. 424, Kluwer Acad. Publ., Dordrecht, 1994, 79-93.

[13] Drozd Yu.A., Ovsienko S.A., Futorny V.M., Irreducible weighted sl(3)-modules, Funct. Anal. Appl. 23 (1989), 217-218.

[14] Drozd Yu.A., Ovsienko S.A., Futorny V.M., On Gel'fand-Zetlin modules, Rend. Circ. Mat. Palermo (2) Suppl. (1991), no. 26, 143-147.

[15] Eckmann B., Schopf A., Über injektive Moduln, Arch. Math. 4 (1953), 75-78.

[16] Feldvoss J., Injective modules and prime ideals of universal enveloping algebras, in Abelian Groups, Rings, Modules, and Homological Algebra, Lect. Notes Pure Appl. Math., Vol. 249, Chapman \& Hall/CRC, Boca Raton, FL, 2006, 107-119, math.RT/0504539.

[17] Fuser A., Autour de la conjecture d'Alexandru, Ph.D. Thesis, Université de Nancy, Nancy, 1997.

[18] Futorny V., Grantcharov D., Ramirez L.E., Singular Gelfand-Tsetlin modules of gl(n), arXiv:1409.0550.

[19] Futorny V., Nakano D.K., Pollack R.D., Representation type of the blocks of category $\mathcal{O}$, Q. J. Math. 52 (2001), 285-305.

[20] Futorny V., Ovsienko S., Kostant's theorem for special filtered algebras, Bull. London Math. Soc. 37 (2005), 187-199, math.RA/0303372.

[21] Futorny V., Ovsienko S., Fibers of characters in Gelfand-Tsetlin categories, Trans. Amer. Math. Soc. 366 (2014), 4173-4208, math.RT/0610071.

[22] Gaillard P.-Y., Introduction to the Alexandru conjecture, math.RT/0003069.

[23] Gel'fand I.M., Tsetlin M.L., Finite-dimensional representations of the group of unimodular matrices, Dokl. Akad. Nauk USSR 71 (1950), 825-828.

[24] Guo X., Liu X., Whittaker modules over generalized Virasoro algebras, Comm. Algebra 39 (2011), 32223231.

[25] Hermann R., Monoidal categories and the Gerstenhaber bracket in Hochschild cohomology, Ph.D. Thesis, Bielefeld University, Germany, 2013, arXiv:1403.3597.

[26] Khomenko O., Some applications of Gelfand-Zetlin modules, in Representations of algebras and related topics, Fields Inst. Commun., Vol. 45, Amer. Math. Soc., Providence, RI, 2005, 205-213.

[27] Khomenko O., Mazorchuk V., Structure of modules induced from simple modules with minimal annihilator, Canad. J. Math. 56 (2004), 293-309.

[28] König S., Mazorchuk V., An equivalence of two categories of $\operatorname{sl}(n, \mathbb{C})$-modules, Algebr. Represent. Theory 5 (2002), 319-329.

[29] Kostant B., On Whittaker vectors and representation theory, Invent. Math. 48 (1978), 101-184.

[30] Krause H., The spectrum of a module category, Mem. Amer. Math. Soc. 149 (2001), x+125 pages.

[31] Mac Lane S., Homology, Classics in Mathematics, Springer-Verlag, Berlin, 1995.

[32] Matlis E., Injective modules over Noetherian rings, Pacific J. Math. 8 (1958), 511-528.

[33] Mazorchuk V., Tableaux realization of generalized Verma modules, Canad. J. Math. 50 (1998), 816-828.

[34] Mazorchuk V., On Gelfand-Zetlin modules over orthogonal Lie algebras, Algebra Colloq. 8 (2001), 345-360.

[35] Mazorchuk V., Quantum deformation and tableaux realization of simple dense $\mathfrak{g l}(n, \mathbb{C})$-modules, J. Algebra Appl. 2 (2003), 1-20.

[36] Mazorchuk V., Ovsienko S., Submodule structure of generalized Verma modules induced from generic Gelfand-Zetlin modules, Algebr. Represent. Theory 1 (1998), 3-26. 
[37] Mazorchuk V., Stroppel C., Cuspidal $\mathfrak{s l}_{n}$-modules and deformations of certain Brauer tree algebras, Adv. Math. 228 (2011), 1008-1042, arXiv:1001.2633.

[38] McDowell E., On modules induced from Whittaker modules, J. Algebra 96 (1985), 161-177.

[39] McDowell E., A module induced from a Whittaker module, Proc. Amer. Math. Soc. 118 (1993), 349-354.

[40] Miličić D., Soergel W., The composition series of modules induced from Whittaker modules, Comment. Math. Helv. 72 (1997), 503-520.

[41] Ondrus M., Whittaker modules, central characters, and tensor products for quantum enveloping algebras, Ph.D. Thesis, The University of Wisconsin, Madison, 2004.

[42] Ondrus M., Wiesner E., Whittaker modules for the Virasoro algebra, J. Algebra Appl. 8 (2009), 363-377, arXiv:0805.2686.

[43] Ondrus M., Wiesner E., Whittaker categories for the Virasoro algebra, Comm. Algebra 41 (2013), 3910-3930, arXiv:1108.2698.

[44] Ovsienko S., Finiteness statements for Gelfand-Zetlin modules, in Third International Algebraic Conference in the Ukraine, Inst. of Math., Kiev, 2002, 323-338.

[45] Ovsienko S., Strongly nilpotent matrices and Gelfand-Zetlin modules, Linear Algebra Appl. 365 (2003), 349-367.

[46] Ramirez L.E., Combinatorics of irreducible Gelfand-Tsetlin sl(3)-modules, Algebra Discrete Math. 14 (2012), 276-296.

[47] Roos J.E., Locally Noetherian categories and generalized strictly linearly compact rings. Applications, in Category Theory, Homology Theory and their Applications, II (Battelle Institute Conference, Seattle, Wash., 1968), Springer, Berlin, 1969, 197-277.

[48] Sevostyanov A., Quantum deformation of Whittaker modules and the Toda lattice, Duke Math. J. 105 (2000), 211-238, math.QA/9905128.

[49] Weibel C.A., An introduction to homological algebra, Cambridge Studies in Advanced Mathematics, Vol. 38, Cambridge University Press, Cambridge, 1994.

[50] Zhelobenko D.P., Compact Lie groups and their representations, Nauka, Moscow, 1970.

[51] Zimmermann A., Representation theory. A homological algebra point of view, Algebra and Applications, Vol. 19, Springer International Publishing, Switzerland, 2014. 